\title{
Influencing Performance Measurements through Varying Packet Capacities of Queue Nodes - DRED
}

\author{
Jafar Ababneh ${ }^{1}$ \\ ${ }^{1}$ The world Islamic science \& education university W.I.S.E, Department of Computer Networks Systems, Jordan \\ Correspondence: Jafar Ababneh, The world Islamic science \& education university W.I.S.E, Department of \\ Computer Networks Systems, Jordan.
}

Received: December 29, 2019

Accepted: March 13, 2020

Online Published: March 16, 2020

doi:10.5539/mas.v14n4p23

URL: https://doi.org/10.5539/mas.v14n4p23

\begin{abstract}
This paper explores the impact of changes to the packet capacities of queue nodes on the performance of congestion algorithms. The dynamic random early drop (DRED) algorithm is crucial in managing congestion, predicting it, and selecting the optimal packet number. Two performance measures were employed to evaluate the effect of varying packet capacities, namely throughput and average queuing delay. Plasticity and efficacy of varying capacities of queue nodes was examined, and varying packet capacities of queue nodes were compared to assess performance. The first queue node performed more effectively than the second queue node in terms of throughput, while the second queue node outperformed the third queue node on both measures.
\end{abstract}

Keywords: average queuing delay, congestion control, DRED, network management and control, packet capacities, queuing network, throughput

\section{Introduction}

In contemporary society, many mission critical systems, as well as the tools many people use in their daily lives, depend on the transmission of massive amounts of data through Wi-Fi networks, Bluetooth, and the Internet of Things (IoT). The rate, at which data passes through these networks, as well as the nature of these networks, has been changing in remarkable ways due to technological advancement. However, with the increasing rate of data transmission, the associated drop off in performance, which arises due to a phenomenon known as congestion, has motivated researchers to find solutions (Fen, 2001; Welzl, 2005).

Algorithms have been developed to target the problem of congestion. These algorithms include (Floyd \& Jacobson's, 1993) RED, ( Aweya et al., 2001) dynamic random early drop (DRED), and (Lapsley \& Low's, 1999) random exponential marking (REM). At the same time, an active area of interest among the research community has been to extend and optimize many of the previously proposed algorithms. For example, (Ott et al., 1999) developed stabilized RED (S-RED), while (Floyd, 2000; Floyd et al., 2001; Feng, 2002) published accounts of gentle RED (G-RED), adaptive RED (A-RED) and blue AQM algorithm, respectively. In the literature, revised version of the DRED congestion algorithm have also been proposed, including (Ababneh et al., 2012; Ababneh et al., 2011; Ababneh et al., April,2010) 3-DRED and (Al-Bahadili, Ababneh \& Thabtah,2011; 2009) mQDRED.

As for the other algorithms that have been developed in more recent years, these include a self-tuning RED algorithm (Jamali et al., 2014), as well as a fuzzy logic gentle random early detection algorithm, which was formulated by (Baklizi et al., 2014). The Markov G-RED, adaptive threshold RED, and fuzzy logic RED algorithms were proposed by (Baklizi et al.,2014), (Patel, 2017), and (Abualhaj et al., 2018), respectively. Other notable algorithms that have relied on advancements in machine learning and neural networks include neural computing fuzzy logic RED (Abualhaj et al., 2018), Enhancing and modifying random early detection made by, (Abu-Shareha, 2019) and (Ahmad Adel, 2019), correspondingly.

A constant in the literature for the past few decades has been the analysis of network performance based on performance measures such as throughput, delay, and packet dropping. However, no previous study has examined the impact of packet size on standard measures of performance. Given this gap in the literature, the purpose of this paper was to assess how an increase in the packet capacities $(k)$ of queue nodes affected throughput $(T j)$ and average queuing delay $(D j)$ in the DRED algorithm. Three nodes were compared in the model to identify which queue node was associated with the greatest increase in performance. 
In terms of the structure and contents of what remains of this paper, the next section presents a review of related work, after which an account of the DRED algorithm is given. In turn, the results of the study are presented and discussed, and concluding remarks are provided.

\section{Related Work}

The purpose of this review is to examine in reverse chronological order the other papers that have been published on the topic of varying parameters regarding the performance measures for DRED of the AQM algorithms.

Various techniques are developed and analyzed for congestion RED, where the early congestion control (ECC) is employed, three sections random early detection (TRED), other methods are based on non-congestion notification, fuzzy logic dimensions, characterization of problems for the congestions in the RED, Hemi-rise cloud model (CRED), and congestion avoidance mechanisms to improve by Learning Automata Like (LAL) philosophy, also several suggestions to improve the congestion control mechanism are presented, (Zala \& Vyas, 2020),

Congestion considered as a most important challenges and critical issue in Wireless sensor networks (WSNs), which affects energy consumption and various parameters of QoS in sensor nodes. Various methods and algorithms employed, and the effective parameter in detecting and controlling congestion is used, (Bohloulzadeh, 2020), the Drop Tail, RED, SFQ, and FQ are assessed by varying the queue size, and the performance analysis and comparison of the various queues are represented in terms of throughput and packet loss, (Patel, N. \& Patel, R. 2020), one-dimensional, discrete-time nonlinear model for Internet congestion control at the routers, which lead to an adaptive congestion control algorithm with a more stable performance than other algorithms currently in use, where the states correspond to the average queue sizes of the incoming data packets, (Amigó, 2020).

Delay-Controller Random Early Detection (DcRED) is proposed to improve network performance under various traffic loads, which gave lesser delay than other algorithms, while maintaining the loss and dropping rates, (Ahmad, 2019), (Babek Abbasov \&, Serdar Korukoğlu,2019) developed an Effective RED to improve RED's performance by reducing packet loss rate, (Baklizi,2019; Baklizi,Jan.,2019) a new management method proposed to Stabilizing Average Queue Length, comprehensive study for multi-criteria evaluation of AQM methods based on current AQM to evaluate criteria conflicting issues and to identify weak points, and possible solutions but criteria significance to be boosted made by (Khatari ,2019). ( Abu-Shareha, 2019) enhanced the performance of the RED algorithm to address the limitations of the original algorithm. The researcher used enhanced random early detection (EnRED) and time-window augmented RED (Windowed-RED). In the study conducted by (Sharma et al., 2018), the researchers proposed P-RED, which constitutes a probabilistic random early detection algorithm for queue management in MANET. Additionally, (Baklizi et al., 2018) examined the FL-RED and AG-RED algorithms, which are both concerned with active queue management. P-Red: Probability based random early detection algorithm for queue management in Manet is presented by, (Sharma, 2017). Others studied nonlinear adjustment for the drop rate at the midpoint between the minimum called Half-Way RED (HRED), (Hamadneh, 2018). An improvement to non-linear RED was formulated by( Zhao et al., 2017), and the researchers achieved this by relying on membership cloud theory, also Patel put forwarded For Adaptive Threshold Based Red(Patel, 2017).

In an earlier paper, (Yu-Hong et al., 2016) published an optimized version of the RED algorithm, referred to as S-RED. Drawing on a three-state Markov-modulated Bernoulli arrival process (MMBP-3), (Baklizi et al., 2016) outlined an efficient modelling of the dynamic G-RED algorithm. (Tsavlidis et al.,2016) assessed a novel router mechanism for networks characterized by selfish flows, while (Baklizi \& Ababneh, 2016) assessed the performance of an enhanced adaptive G-RED algorithm in a variety of congestion scenarios. (Baklizi et al., 2014) published a paper in which fuzzy logic was used to manage and control the G-RED algorithm, and the method relied on the delay rate and average queue length.

The study conducted by (Ababneh et al., 2012; Ababneh et al., 2011;Ababneh et al., April 2010; Ababneh et al., 2010; Al-Bahadili, Ababneh \& Thabtah, 2009) formulated 3-DRED and MQDRED, while (Abdel Jaber et al 2011; Abdel Jaber et al., 2008) proposed a DRED algorithm involving a pair of queue nodes for congestion control. (Abdeljaber, et al., 2012) investigated performance of a number of Active Queue Management Techniques, (Ariba et al., 2008) outlined an AQM design to reveal the usefulness of the design in mitigating congestion. As for the previous study of (Hoflack et al., 2008), the researchers studied file server traffic and derived an approximation calculation for the buffer occupancy's tail probabilities. The autonomous RED (AU-RED) algorithm devised by (Ho \& Lin, 2008) was notable due to the way it allowed more elasticity to put up for various situations. In (Walraevens et al.'s, 2008) paper, the researchers analyzed a discrete-time priority 
queue, in which session-based arrivals were a feature.

As for the other relevant studies undertaken in this area, (Wang et al., 2008) investigated the impact on AQM performance of the squared coefficient of variation of specific types of traffic.In this area, (Abdel-Jaber et al., 2007) conducted a notable research project that resulted in favorable performance matrices when compared to other studies. (Byun \& Baras, 2007) proposed a novel adaptive virtual queue RED algorithm (AVQ-RED), the purpose of which was to enhance performance, to maintain a high and stable level of link utilization, to minimize queuing delay, and to mitigate high consecutive packet drop rates.

To account for the impact on a range of performance measures made by modifications to given input values, (Guan et al., 2007) used a two-dimensional discrete-time Markov chain. In (Le et al.'s,2007), studies, the researchers used various types of controllers, including a random exponential marking (REM) controller and a proportional integral (PI) controller, to investigate performance measures. Alongside this, the researchers drew on the A-RED algorithm as the focus of their analysis. They demonstrated that characteristics of the algorithm were improvements in transient-state and steady-state performance. (Yamagaki et al., 2007) provided an outlined of dual metrics fair queuing (DMFQ), the purpose being to improve the quality of network service. (Wang et al.'s, 2007) loss ratio-based RED (L-RED), which is a novel AQM algorithm, was proposed in relation to queue length, and the purpose of the algorithm was to adjust the likelihood of packet drop in an adaptive manner.

A range of related studies were published in 2006, including (Awan et al., 2006) and (Wu et al., 2006), among others. In the first of these studies, the researchers proposed and evaluated a structure based on blocking under AQM scheme, the purpose of which was to examine queuing network performance. In the second study, namely (Wu et al., 2006), the researchers designed server fairness RED (SF-RED), while the third study, (Wang et al. 2006), proposed an analytical performance model relying on the quantity of queued packets. A range of performance measures were derived from the research project, including system throughput, mean waiting time, mean queuing length, likelihood of packet loss, and response time. Also in 2006, Kim and Park proposed a wavelet neural network (WNN) controller for AQM in the context of an end-to-end TCP network. Note worthily, the controller was grounded in the adaptive learning rate (ALR) method. The final notable study published in 2006 was that of (Kulkarni et al.,2006) which proposed PAQMAN, an AQM based on proactive prediction.

In 2005, Chen outlined a general functional optimization model for the design of AQM schemes (Chen, 2005), while (Cho et al.) formulated an AQM method relying on dynamic neural networks. In the latter study, the researchers relied on the use of back-propagation for queue size regulation, which allowed them to optimize TCP performance. Another study conducted in 2005, namely Gao et al., sought to maximize relevant network performance measures relating to the forecasting of network traffic, and this led to the development of the PFED algorithm. Additionally, (Ku et al, 2005) devised a technique by which the packet-loss ratio and end-to-end delay could be minimized, while (Kumar \& Mahapatra, 2005) improved throughput and average delay. The latter study relied on the ISMM algorithm, which is concerned with scheduling and memory management.

(Aweya \& Ouellette, 2007) extended DRED to permit the prioritization of network traffic with multiple packet drop precedence. As for the study conducted by (Joshi et al., 2004), the researchers sought to address network congestion by examining multiple average-multiple threshold (MAMT). In order to facilitate effective control of packet losses without undermining link utilization, (Aweya et al., 2003) assessed the AQM scheme.

Soon after the turn of the new millennium, several notable studies were published in this area. For example, ( Joo et al., 2003) proposed a novel AQM algorithm intended to improve performance based on a simple process of choosing parameters for steady and other load changes. (Fen et al., 2002) derived and assessed stochastic fair BLUE (SFB), the purpose being to detect and rate-limit unresponsive flows with the minimal level of state information. Finally, (Wydrowski et al. 2002) devised a novel AQM algorithm referred to as the generalized random early evasion network (GREEN) in order to increase network performance measures, while (Aweya et al., 2001) highlighted how a specific method could be used to increase the efficacy of RED schemes.

Having surveyed related work from 2001 until the present day, it is worth emphasizing that each of the proposed mechanisms, schemes, models, and algorithms used by routers and switches in a network have contributed to vast improvements in the control, management, and optimization of performance measurements for network quality of service. Nevertheless, although extensive assessments of throughput, packet-loss rate, average queuing delay, and other measures of performance have been undertaken, no previous study has examined the effect of router size on quality of service for each of these performance measures. 


\section{DRED Algorithm}

In (Aweya's, 2001) study, the DRED algorithm was proposed to address the network congestion algorithm. In contrast to RED, which drops every packet that arrives, DRED relies on the packet probability dropping (Lapsley \& Low, 1999; Chen \& Yang, 2009). Below, equations (1), (2), and (3) indicate the capacity of the DRED router buffer $(k)$, while the thresholds $\mathrm{H} 1, \mathrm{H} 2$, and $\mathrm{H} 3$ are expressed in equation (4). The calculations given here depend on the queuing network system given in Figure 1 (Ababneh et al., 2010).

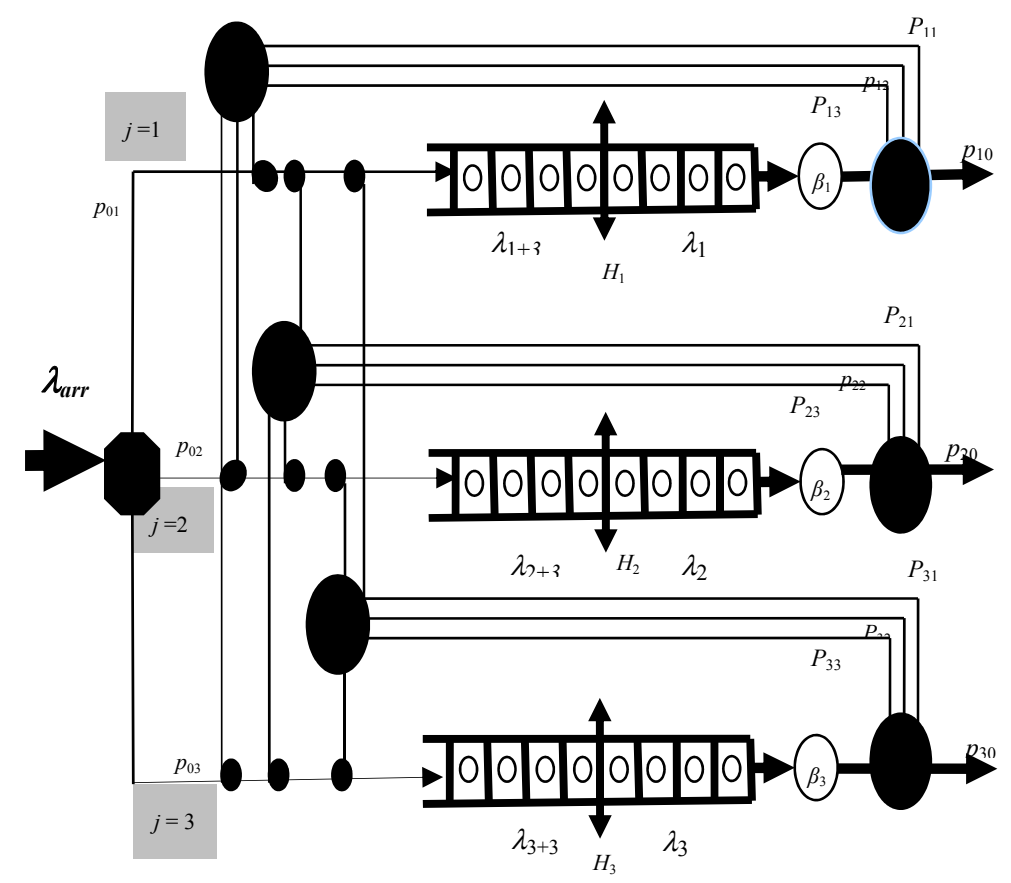

Figure 1. Queuing network system with

The abovementioned equations are given as follows:

$$
\begin{gathered}
E(t)=Q(t)-Q t a r \\
F(t)=F(t-1)(1-w q)+w q E(t) \\
\left.p_{d}(t)=\min \left\{\max \left(p_{d}(t-1)+\epsilon(F(t) / K), 0\right)\right), 1\right\} \\
H_{j}=[0.9 K / 2] \text { for } j=1,2,3
\end{gathered}
$$

In the following, equation (5) expresses the calculation for throughput $\left(\mathrm{T}_{j}\right)$, while equation (6) shows how to compute average queuing delay $(\mathrm{D} j)$.

$$
\begin{gathered}
T_{j}=\beta_{j} \sum_{i=1}^{K} \Pi_{i}=\beta_{j}\left(1-\Pi_{0}\right) \\
D_{j}=\left[Q_{\text {avgj }} / T_{j}\right] \text { slots }=\left[P^{(1)}(1) / T_{j}\right] \text { slots }=\left[\sum_{i=0}^{K_{j}} i \Pi_{i} / T_{j}\right] \text { slots }
\end{gathered}
$$

Table 1 provides an overview of the parameters employed for the congestion metric regarding the DRED algorithm. 
Table 1. Parameters for DRED

\begin{tabular}{cc}
\hline Parameter & Value \\
\hline Units of time $(t)$ & $\begin{array}{c}\text { The time taken to send } 10 \text { packets or, } \\
\text { as an alternative, another suitable value }\end{array}$ \\
Target level for the queue $\left(Q_{\text {avgt }}\right)$ & $Q_{\text {avgt }}=0.5 \mathrm{k}$ \\
Indication congestion threshold & $H=0.9 Q_{\text {avgt }}$ \\
Buffer capacity & $\mathrm{K}$ \\
Queue weight $\left(Q_{w}\right)$ & $0.2 \%$ \\
System control $(\epsilon)$ & $0.005 \%$ \\
\hline
\end{tabular}

Regarding the identical Bernoulli process, which - notably - is also distributed in an independent way, this is $a_{n} \in\{0,1\}, n=0,1,2,3 \ldots$, and it is used in this paper. Furthermore, $\lambda_{i}(j=1,2,3)$ is the probability of a packet arriving at the router buffer of the three queue nodes. In the case of $\beta_{j}$, this denotes the likelihood that the packet will exit in a slot from the nodes. The assumption is also made that the queueing network is not in disequilibrium, and that the $Q_{a v g}$ process for every queue node can be formalised as a Markov chain characterised by finite state spaces. Regarding the state spaces for the queue node $(j)$, this can be expressed as $\left\{0,1,2,3, \ldots, H_{j}-1, H_{j}, H_{j}+1, \ldots, K_{j}-1, K_{j}\right\}$.

\section{Results and Discussion}

In our experiments, a range of parameters were used, and we also considered the priority of principles where the first queue node was associated with a greater priority than the second, and the second greater than the third. As such, this priority was transitive, in that the first queue node had a greater priority than the third regarding the serving of packets routed external to the network. The impact of varying the packet capacities (buffer size) of the queue nodes on various measures of performance was examined (namely, throughput and average queuing delay) in order to determine which one lead to the highest quality of service.

Tables 2, 3, and 4 present the results of varying queue capacities (threshold) and the performance measures for each queue node in our model. The parameters for the first node were $\mathrm{r} 01=0.50$, which refers to the likelihood of the packets being routed external to the network; and for $\lambda_{1}, \beta_{1}$, and $K_{1}$, the values were $0.75,0.90$, and $[12,20,40,80,160,320,640]$, respectively. Additionally, the routing probabilities associated with the first queue node, namely $\mathrm{r}_{10}, \mathrm{r}_{11}, \mathrm{r}_{12}$, and $\mathrm{r}_{13}$, were $0.4,0.3,0.2$, and 0.1 , respectively. The parameters for second node were $\mathrm{r} 02=0.30$, and $\lambda_{2}, \beta_{2}$, and $K_{2}$ were $0.75,0.90$, and $[12,20,40,80,160,320,640]$, respectively. For $\mathrm{r}_{20}, \mathrm{r}_{21}, \mathrm{r}_{22}$, and $r_{23}$, the values were $0.4,0.3,0.2$, and 0.1 , respectively. Finally, for the third node, $\mathrm{r} 03=0.30, \lambda_{3}, \beta_{3}$, and $K_{3}$ were $0.75,0.90$, and $[12,20,40,80,160,320,640]$, respectively, and $\mathrm{r}_{30}, \mathrm{r}_{31}, \mathrm{r}_{32}$, and $\mathrm{r}_{33}$ were $0.4,0.3$, 0.2 , and 0.1 , respectively.

Table 2. Performance measures $\left(\mathrm{T}_{1}, \mathrm{D}_{1}\right)$ results of first node with different queue sizes $(K)$

\begin{tabular}{ccc}
\hline$K$ & $T_{1}$ & $D_{l}$ \\
\hline 12 & $8.6446 \mathrm{E}-01$ & $2.7573 \mathrm{E}+00$ \\
20 & $8.7196 \mathrm{E}-01$ & $3.7996 \mathrm{E}+00$ \\
40 & $8.7471 \mathrm{E}-01$ & $4.7700 \mathrm{E}+00$ \\
80 & $8.7500 \mathrm{E}-01$ & $4.9950 \mathrm{E}+00$ \\
160 & $8.7500 \mathrm{E}-01$ & $5.0000 \mathrm{E}+00$ \\
320 & $8.7500 \mathrm{E}-01$ & $5.0000 \mathrm{E}+00$ \\
640 & $8.7500 \mathrm{E}-01$ & $5.0000 \mathrm{E}+00$ \\
\hline
\end{tabular}


Table 3. Performance measures $\left(\mathrm{T}_{2}, \mathrm{D}_{2}\right)$ results of first node with different queue sizes $(K)$

\begin{tabular}{ccc}
\hline$K$ & $T_{2}$ & $D_{2}$ \\
\hline 12 & $5.6011 \mathrm{E}-01$ & $1.2967 \mathrm{E}+00$ \\
20 & $5.6000 \mathrm{E}-01$ & $1.2941 \mathrm{E}+00$ \\
40 & $5.6000 \mathrm{E}-01$ & $1.2941 \mathrm{E}+00$ \\
80 & $5.6000 \mathrm{E}-01$ & $1.2941 \mathrm{E}+00$ \\
160 & $5.6000 \mathrm{E}-01$ & $1.2941 \mathrm{E}+00$ \\
320 & $5.6000 \mathrm{E}-01$ & $1.2941 \mathrm{E}+00$ \\
640 & $5.6000 \mathrm{E}-01$ & $1.2941 \mathrm{E}+00$ \\
\hline
\end{tabular}

Table 4. Performance measures $\left(\mathrm{T}_{3}, \mathrm{D}_{3}\right)$ results of first node with different queue sizes $(K)$

\begin{tabular}{ccc}
\hline$K$ & $T_{3}$ & $D_{3}$ \\
\hline 12 & $3.1500 \mathrm{E}-01$ & $7.0521 \mathrm{E}-01$ \\
20 & $3.1500 \mathrm{E}-01$ & $7.1718 \mathrm{E}-01$ \\
40 & $3.1500 \mathrm{E}-01$ & $7.1719 \mathrm{E}-01$ \\
80 & $3.1500 \mathrm{E}-01$ & $7.1719 \mathrm{E}-01$ \\
160 & $3.1500 \mathrm{E}-01$ & $7.1719 \mathrm{E}-01$ \\
320 & $3.1500 \mathrm{E}-01$ & $7.1719 \mathrm{E}-01$ \\
640 & $3.1500 \mathrm{E}-01$ & $7.1719 \mathrm{E}-01$
\end{tabular}

Figures 2 and 3 present the effect of varying the packet size on throughput and average queuing delay. Since Figure 2 indicates a relationship between varying packet size and throughput, it is reasonable to conclude that, for the three nodes, the throughput increased slightly (less than 7.4938E-03), after which it plateaued for the other queue sizes. In the case of Figure 3, no increase in queue nodes two and three was observed due to the delay when the buffer size was increased and the traffic load was fixed. However, the delay associated with the first queue node increased until the buffer size reached 80 , which can be attributed to the fact that it had the highest routing priority of routing probability when compared to the other nodes.

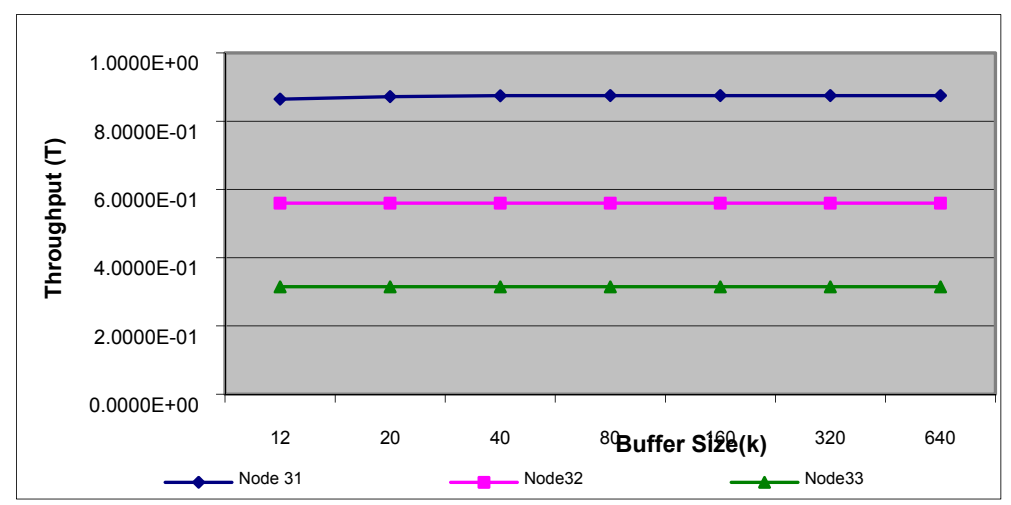

Figure 2. k VS T 


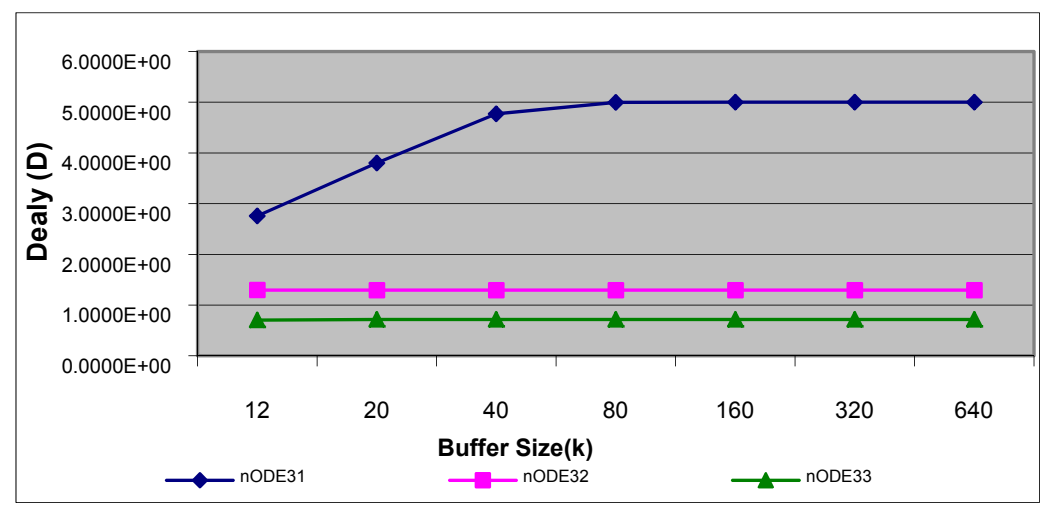

Figure 3. k VS D

Setting a buffer size of less than 20 is justified due to the results of the performance measure, combined with the fixed arrival packet rate and the routing probability. The threshold varied because equation (3) demonstrated that a linear relationship existed between buffer size and threshold, which is linked to the moderate probability of packet arrival $\left(\lambda_{\text {arr }}=0.75\right)$.

\section{Conclusion}

The purpose of this paper was to investigate the effect of varying packet capacities (buffer size) of queue nodes on throughput and average queuing delay, thereby indicating which one was associated with the greatest network quality of service. Three nodes were compared to determine which was associated with the most favorable results, and node performance was measured by setting various parameters. Regarding the relationship between varying packet size and throughput, the results indicated that, for each of the nodes examined in this paper, throughput increased slightly, and then froze to a fixed value. Additionally, regarding the link between varying packet size and average queuing delay, only the first node increased, which can be attributed to its higher priority. Future studies should examine the impact of packet size variety on the probability of packet arrival, particularly the highest packet arrival. This should be examined for different algorithms, thereby facilitating a comparative examination.

\section{References}

Ababneh, J., Abdel-jaber, H., Albalas \& F., Daoud, A. (2012). Simulation in Computer Network Design and Modeling: Use and Analysis, Analyzing and Evaluating Current Computer Networks Simulation Models, 459- 478, https://doi.org/10.4018/978-1-4666-0191-8.ch022.

Ababneh, J., Thabtah, F., Abdel-Jaber, H., Hadi, W. \& Badarneh, E. (2011). Evaluating the Performance of Active Queue Management Using Discrete-Time Analytical Model, Technology Engineering and Management in Aviation: Advancements and Discoveries, Edition: 1, Chapter: 18, Publisher: IGI, 308-324, https://doi.org/10.4018/978-1-60960-887-3.

Ababneh J., Abdeljaber H., Thabtah F., Hadi W. \& Badarneh E. (April,2010). Derivation of Three Queue Nodes Discrete-Time Analytical Model Based on DRED Algorithm. The Seventh IEEE International Conference on Information Technology: New Generations (ITNG 2010). IEEE Computer Society, 885-890, April 2010, Las Vegas, USA, https://doi.org/10.1109/ITNG.2010.252.

Ababneh, J., Thabtah, F., Abdeljaber, H., Hadi, W. \& Badarneh, E. (March, 2010). Discrete-time analytical model for evaluating the performance of three queue nodes based on dynamic RED algorithm. Aviation Information Technology, Engineering and Management AITEM2010), 152-163.

Abdeljaber, H., Thabtah,F., Daoud,A, Ababneh,J. 1 \& Baklizi, M. (2012). Performance Investigations of Some Active Queue Management Techniques Using Simulation, International Journal on New Computer Architectures and Their Applications (IJNCAA) 2(1), 286-301 The Society of Digital Information and Wireless Communications, (ISSN: 2220-9085)

Abdeljaber, H. ,M. Woodward, F. Thabtah \& Etbega, M. (2007). A Discrete-Time Queue Analytical Model based on Dynamic Random Early Drop, Proceedings of the 4th IEEE International Conference on Information Technology: New Generations (ITNG 2007). Las Vegas, USA, 71-76. 
Abdeljaber, H., F. Thabtah \& M. Woodward, (2008). Performance Measures Evaluation for DRED Discrete-Time Queuing Network Analytical Model. Proceedings of the Journal of Network and Computer Applications, 31(4), 750-770, https://doi.org/10.1142/S1793962311500048.

Abdel-Jaber, H., J. Ababneh, F. Thabtah, A. M. Daoud \& M. Baklizi, (2011). Performance Analysis Of The Proposed Adaptive Gentle Random Early Detection Method Under Noncongestion And Congestion Situations, International Conference On Digital Enterprise And Information Systems,London, Uk,2011, 592-603. International Journal of Computer Networks \& Communications, 11(6), November 2019, https://doi.org/10.1007/978-3-642-22603-8_52.

Abualhaj, M. M., A.A. Abu-Shareha \&Al-Tahrawi, M.M. (2018). FLRED: An Efficient Fuzzy Logic Based Network Congestion Control Method. Neural Computing and Applications, 30(3), 925-935, https://doi.org/10.1007/s00521-016-2730-9.

Abu-Shareha, A.A. (2019). Enhanced Random Early Detection Using Responsive Congestion Indicators. International Journal of Advanced Computer Science and Applications, 10(3), 358-367, https://doi.org/ 10.5121/ijcnc.2019.11604.

Ahmad Adel, (2019). Controlling Delay at the Router Buffer using Modified Random Early Detection International, 11(6), 63-75, https://doi.org/10.5121/ijenc.2019.11604.

Al-Bahadili, H., Ababneh, J. \& Thabtah, F. (2011). Analytical modeling of a multi-queue nodes network router. Int. J. Autom. Compute, 8, 459-464, https://doi.org/10.1007/s11633-011-0604-2.

Al-Bahadili,H, Ababneh,J \& Thabtah,F, (2009). Derivation of an analytical model for evaluating the performance of a multi-queue nodes network router, ISIICT09: Proceedings of the Third international conference on Innovation and Information and Communication Technology, Amman, Jordan, 8, https://doi.org/10.5555/2228029.2228040.

Amigó, M. José;Duran, Guillem;Giménez, Angel; Martínez-Bonastre \& Oscar; Valero, José, (2020). Generalized TCP-RED dynamical model for Internet congestion control. Communications in Nonlinear Science and Numerical Simulation, 82, https://doi.org/10.1016/j.cnsns.2019.105075.

Ariba, Y. \& F. Gouaisbaut, (2008). Construction of Lyapunov-Krasovskii functional for time-varying delay systems, 2008 47th IEEE Conference on Decision and Control, Cancun, 3995-4000.

Awan, I., Yar, A. \& Woodward (2006). Analysis of Queuing Networks with Blocking under Active Queue Management Scheme, proceedings of the 12th International Conference on Parallel and Distributed Systems (ICPADS06). Vol. 2, 61-68.

Aweya, J., M. Ouellette \& D. Y. Montuno, (2007). A simple mechanism for stabilizing network queues in TCP/IP networks. International Journal of Network Management archive, 17(4), 275 - 286, https://doi.org/10.1002/nem.625.

Aweya, J. M. Ouellette \& Montuno, D. Y. (2003). Active queue management with flow proportional buffering. journal of Computer Networks, 13(3), 211-229, https://doi.org/10.1002/nem.479.

Aweya, J. , M.Ouellette ,D. Y. Montuno \& Chapman, A., (2001). An adaptive buffer management mechanism for improving TCP behavior under heavy loads, ICC 2001. IEEE International Conference, 10, 3217 - 3223.

Babek Abbasov \& Serdar Korukoğlu, (2019). Effective RED: An algorithm to improve REDs performance by reducing packet loss rate. Journal of Network and Computer Applications, 32(3), 703-709, https://doi.org/10.1016/j.jnca.2008.07.001.

Baklizi, Mahmoud. (Jan.,2019). FLACC: Fuzzy Logic Approach for Congestion Control. International Journal of Advanced Computer Science and Applications. https://doi.org/10.14569/IJACSA.2019.0100707.

Baklizi, M. (2019). Stabilizing Average Queue Length in Active Queu Management Method. International Journal of Advanced Computer Science and Applications, 10, https://doi.org/10.14569/IJACSA.2019.0100310.

Bakliz, M., Ababneh,J. \& Abdallah, N. (2018). PERFORMANCE INVESTIGATIONS OF FLRED AND AGRED ACTIVE QUEUE MANAGEMENT METHODS,Journal of Theoretical and Applied Information Technology 31st October 2018. Vol.96. No 20 (C) 2005 - ongoing JATIT \& LLS ISSN: 1992-8645 www.jatit.org E-ISSN: 1817-3195 6697 Proceedings of Academicsera 13 th International Conference, Istanbul, Turkey, 2018.

Bakliz, M. \& Ababneh,J. (2016). Performance Evaluation of the Proposed Enhanced Adaptive Gentle Random Early Detection Algorithm in Congestion Situations. International Journal of Current Engineering and 
Technology, 6(5), 1658-1664.

Baklizi,M. \& Ababneh,J. (2016). A Survey in Active Queue Management Methods According to Performance Measures. International Journal of Computer Trends and Technology (IJCTT), 37(3), 145-152, www.ijcttjournal.org. Published by Seventh Sense Research Group, https://doi.org/10.14445/22312803/IJCTT-V38P126.

Baklizi,M., H. Abdel-Jaber, A. A. Abu-Shareha, M. Abualhaj \& S. Armadas, (2014). Fuzzy Logic Controller Of Gentle Random Early Detection Based On Average Queue Length And Delay Rate. International Journal of Fuzzy Systems, 16(1), 9-19.

Bohloulzadeh, A. \& Rajaei, M. (2020). A Survey on Congestion Control Protocols in Wireless Sensor Networks. Int J Wireless Inf Networks. https://doi.org/10.1007/s10776-020-00479-3.

Byun, D., Baras, J. (2009). Adaptive Virtual Queue Random Early Detection in Satellite Networks, 63-82, https://doi.org/10.1007/978-0-387-71787-6_4.

Chen,W. \& Yang, H. (2009). The mechanism of adapting RED parameters to TCP traffic, Computer Communications, 32(13-14)1525-1530, https://doi.org/10.1016/j.comcom.2009.05.008

Chen, Yixin, (2005). Functional Optimization Models for Active Queue Management, Department of Computer Science and Engineering Washington University in St Louis, USA, Dec https://doi.org/10.1.1.111.3416.

Feng, W., D. Kandlur, D. Saha \& K. Shin, (2002). The BLUE active queue management algorithms. IEEE/ACM Transactions on Networking, 10(4), 513-528, Aug. 2002, https://doi.org/10.1109/TNET.2002.801399.

Feng, W. C., Kandlur, D. D., Saha, D. \& Shin, K. G. (2001, April). Stochastic fair blue: A queue management algorithm for enforcing fairness. In Proceedings IEEE INFOCOM 2001. Conference on Computer Communications. Twentieth Annual Joint Conference of the IEEE Computer and Communications Society (Cat. No. 01CH37213) (Vol. 3, pp. 1520-1529). IEEE.

Floyd, S.G. Ramakrishna \& S. Shenker, (2001). Adaptive RED: An Algorithm for Increasing the Robustness of REDs Active Queue Management, Technical report, ICSI.

Floyd, S. (2000). Recommendations on using the gentle variant of RED, Available at http://www.aciri.org/floyd/red/gentle.html.

Floyd, S. \& V. Jacobson, (1993). Random Early Detection Gateways for Congestion Avoidance. IEEE/ACM Transactions on Networking, 397- 413, https://doi.org/10.1109/90.251892.

Guan, L., I. Awan, M. Woodward \& X. Wang (2007). Discrete-time performance analysis of a congestion control mechanism based on RED under multi-class bursty and correlated traffic. Journal of Systems and Software, 80(10), 1716-1725.

Hamadneh.N., Obiedat, M.amoon \& Qawasmeh, A. (2018). HRED, An Active Queue Management Approach for The NS2 Simulator. Recent Patents on Computer Science, 12(3), https://doi.org/10.2174/2213275912666181205155828.

Ho, H.\& Lin, W. (2008). AURED:Autonomous Random Early Detection for TCP Congestion Control, 0,79-84, $\mathrm{JO}$ - Systems and Networks Communication, International Conference on, https://doi.org/10.1109/ICSNC.2008.22.

Hoflack L., De Vuyst S., Wittevrongel S. \& Bruneel H. (2008). Modeling Web Server Traffic with Session-Based Arrival Streams. In: Al-Begain K., Heindl A., Telek M. (eds) Analytical and Stochastic Modeling Techniques and Applications. ASMTA 2008. Lecture Notes in Computer Science, vol 5055. Springer, Berlin, Heidelberg, https://doi.org/10.1007/978-3-540-68982-9_4

Jamali, S., Alipasandi, N. \& Alipasandi, B. (2014). An Improvement over Random Early Detection Algorithm: A Self-Tuning Approach, Computer Engineering Department. University of Mohaghegh Ardabili,jesel, 2(2). https://doi.org/10.22061/JECEI.2014.242.

Joo, C., S. Bahk, Steven S. \& Lumetta. (2003). Hybrid Active Queue Management, Proceedings of the Eighth IEEE International Symposium on Computers and Communication (ISCC03). IEEE, 2, 999- 1004.

Joshi, M., Mansata, A., Talauliker, S. \& Beard, C. (2005). Design and analysis of multi-level active queue management mechanisms for emergency traffic. Computer Communications, 28(2), 162-173.

Khatari, Maimuna, Zaidan, A., Zidan, B., Albahri, O.s, Alsalem \& Mohammed, (2019). Multi-Criteria Evaluation and Benchmarking for Active Queue Management Methods: Open Issues, Challenges and 
Recommended Pathway Solutions. International Journal of Information Technology \& Decision Making, 18(1). https://doi.org/10.1142/S0219622019300039.

Ku,C. Sao-Jie Chen, Jan-Ming Ho \& Ray-I Chang, (2005). end-to-end performance by active queue management, Advanced Information Networking and Applications. 19th International Conference on Publication, 2, $337-$ 340, https://doi.org/10.1109/AINA10302.2005.

Kulkarni, P. G., McClean, S. I., Parr, G. P. \& Black, M. M. (2006, April). Proactive predictive queue management for improved QoS in IP networks. In International Conference on Networking, International Conference on Systems and International Conference on Mobile Communications and Learning Technologies (ICNICONSMCL'06) (pp. 7-7). IEEE. https://doi.org/10.1109/ICNICONSMCL.2006.175.

Kumar Anuj \& Rabi N. Mahapatra (2005). Integrated scheduling and buffer management input queued switches with finite buffer space. Elevier, 29(1), 42-51, Computer Communications, https://doi.org/10.1016/j.comcom.2005.03.006

Lapsley, D. \& Low, S. (1999, December). Random early marking for Internet congestion control. In Seamless Interconnection for Universal Services. Global Telecommunications Conference. GLOBECOM'99. (Cat. No. 99CH37042) (Vol. 3, pp. 1747-1752). IEEE.

Lapsley, D. \& S. Low, (1999). Random Early Marking: An Optimization Approach to Internet Congestion Control, in Proceedings of IEEE ICON 99, https://doi.org/10.1007/978-1-4757-3762-2.

van Leeuwaarden, J., Denteneer, D. \& Resing, J. (2006). A discrete-time queueing model with periodically scheduled arrival and departure slots. Performance Evaluation, 63(4-5), 278-294.

Ott, T., T. Lakshman \& L. Wong (1999). SRED: Stabilized RED, in Proc. IEEE INFOCOM, Mar., 1346-1355.

Patel N. \& Patel R. (2020). Performance Analysis of Network with Different Queuing Mechanisms in TCP/FTP and UDP/FTP Scenario. In: Somani A., Shekhawat R., Mundra A., Srivastava S., Verma V. (eds) Smart Systems and IoT: Innovations in Computing. Smart Innovation, Systems and Technologies, vol 141. Springer, Singapore, https://doi.org/10.1007/978-981-13-8406-6_2.

Patel, Z. M. (2017). Queue Occupancy Estimation Technique for Adaptive Threshold Based Red. Ieee International Conference on Circuits And Systems (Iccs). Thiruvananthapuram, Kerala, India., 437-440.

Sharma, N. S. S. Rajput, A. K. Dwivedi \& M. Shrimali, (2017). P-Red: Probability Based Random Early Detection Algorithm for Queue Management. Manet, Advances in Computer And Computational Sciences, 637-643.

Tsavlidis, L., Efraimidis, P. S. \& Koutsiamanis, R. A. (2016). Prince: an effective router mechanism for networks with selfish flows. Journal of Internet Engineering, 6(1), 355-362.

Walraevens,j., Sabine Wittevrongel \& Herwig Bruneel, (2008). Analysis of Priority Queues with Session-Based Arrival Streams, Seventh International Conference on Networking, ICN, 503-510, https://doi.org/10.1109/ICN.2008.47.

Wang, L. Geyong Min \& Awan, I. (2008). An Analytical Model for Priority-Based AQM in the Presence of Heterogeneous Network Traffic, Advanced Information Networking and Applications, AINA 2008. 22nd International Conference on22nd International Conference on, 93-99, 2008, https://doi.org/10.1109/AINA.2008.140.

Wang, C. Liu, J., AU - Li, B., Sohraby, K.azem \& Hou, Y.iwei,(2007). LRED: A Robust and Responsive AQM Algorithm Using Packet Loss Ratio Measurement, Parallel and Distributed Systems. IEEE Transactions, 18, 29- 43, https://doi.org/10.1109/TPDS.2007.253279.

Wang, L., Geyong Min \& Irfan Awan, (2006). Modeling active Queue Management with Different Traffic Classes, aina. 20th International Conference on Advanced Information Networking and Applications, 2, 442-446.

Wydrowski, B. \& Zukerman,M. (2002). GREEN: An Active Queue Management Algorithm for a self-Managed Internet. Proceedings of ICC 2002, 4, 2368-2372.

Wu, H., Wu, C. \& Lin, W. (2006). SF-RED - A novel server-based AQM to provide inter-server fairness service, (1), 501-506, https://doi.org/10.1109/ICPADS.2006.99.

Yu-hong, Z. TU \& Xu-yan(2016). International Journal of u- and e- Service, Science and Technology. Improved Way of RED Algorithm S-RED, 9(2), 375-384 
Yamagaki, N. Hideki Tode \& Koso Murakami (2005). DMFQ: Hardware Design of Flow-Based Queue Management Scheme for Improving the Fairness, IEICE Transactions,88-B,413-1423.

Zala D.D. \& Vyas A.K. (2020). Comparative Analysis of RED Queue Variants for Data Traffic Reduction Over Wireless Network. In: Mehta A., Rawat A., Chauhan P. (eds) Recent Advances in Communication Infrastructure. Lecture Notes in Electrical Engineering, 618. Springer, Singapore, https://doi.org/10.1007/978-981-15-0974-2_3.

\section{Copyrights}

Copyright for this article is retained by the author(s), with first publication rights granted to the journal.

This is an open-access article distributed under the terms and conditions of the Creative Commons Attribution license (http://creativecommons.org/licenses/by/3.0/). 